\title{
Evaluation of the novel coronavirus disease in Turkish Children; Preliminary Outcomes
}

\author{
kamil YILMAZ ${ }^{1}$, Ayfer Gozu Pirinccioglu ${ }^{1}$, Fesih Aktar ${ }^{1}$, alper akin ${ }^{2}$, Müsemma Karabel $^{3}$, \\ ilyas yolbas ${ }^{4}$, Sabahattin Ertugrul ${ }^{3}, \mathrm{~V} . \mathrm{Uzel}^{5}$, Edip Unal ${ }^{1}$, Ibrahim Deger ${ }^{1}$, Kadri Toy $^{1}$, \\ Hasan Zan ${ }^{1}$, Cihan Onder ${ }^{1}$, Recep Tekin ${ }^{1}$, Cigdem Mermutluoglu ${ }^{1}$, E. Yılmaz ${ }^{1}$, Hadice \\ Selimoglu Sen ${ }^{6}$, Sureyya Yılmaz ${ }^{1}$, Cihan Ozmen ${ }^{1}$, Velat Şen ${ }^{5}$, and Zulfikar Yılmaz ${ }^{1}$ \\ ${ }^{1}$ Dicle Üniversitesi \\ ${ }^{2}$ Dicle University \\ ${ }^{3}$ Dicle University School of Medicine \\ ${ }^{4}$ dicle university \\ ${ }^{5}$ Dicle Üniversity \\ ${ }^{6}$ Dicle University Medical Faculty
}

July 13, 2020

\begin{abstract}
Background: The novel coronavirus disease (Covid-19) can progress with mild to moderate or self-limiting clinical findings in children. The aim of this study was to investigate the disease features of Covid-19 in Turkish children. Methods: Children diagnosed by the method of RT-PCR for Covid-19 at the Dicle University Department of Pediatric, between April and June 2020, were evaluated. Hospital records were investigated retrospectively. Results: One hundred and five patients children with the mean age of $108.64 \pm 65.61$ were enrolled in this study. The most common cause of transmission in pediatric patients was contacting with a family member diagnosed with COVID-19 $(\mathrm{n}=91,86.7 \%)$. The most common admission complaints were dry cough $(n=17,16.2 \%)$, fever $(n=16,15.2 \%)$, lassitude and fatigue $(n=14,13.3 \%)$ respectively. More than $95 \%$ of all children with Covid-19 had asymptomatic, mild, or moderate cases. CRP was identified only independent factor associated with long duration of hospitalization. Conclusion: The results of this study show preliminary results of a study investigating the effect of Covid-19 on Turkish children. A clear understanding of the local epidemiology of corona virus infections and identification of risk factors is critical for the successful implementation of the prevention and control program.
\end{abstract}

\section{Introduction}

Coronaviruses (CoVs) are the single-chain, positive-stranded and enveloped RNA viruses that related to human disease had been determined (1). The virus responsible for novel coronavirus disease (COVID-19) is located under the Sarbecovirus subspecies in the genus betacoronavirus, which it contains in severe acute respiratory syndrome coronavirus (SARS-CoV) and Middle East respiratory syndrome coronavirus (MERS$\mathrm{CoV}$. The new nomenclature of the virus has been accepted as severe acute respiratory syndrome coronavirus 2 (SARS-CoV-2) (2).

Among the coronaviruses causing disease in humans, HCoV 229E, NL63, OC43, and HKU1 are the most frequently isolated. Despite all of the coronaviruses can infect subjects of all ages, these subtypes are the cause of endemics of upper respiratory tract infections in adults. However, many coronavirus subtypes can cause severe disease settings in humans $(3,4,5)$.

Coronaviruses are capable of mutation leading to novel CoVs that can be transmitted from animals to 
humans. At first, SARS-CoV is transmitted from bearcats to humans in 2002 and resulted hundreds of people to lose their lives in 37 countries. After 10 years, in 2012 MERS-CoV, was first described in humans which was transmitted from dromedary camels to humans and caused 858 fatalities $(6,7)$.

In December 2019, the novel SARS-CoV-2 was reported in Wuhan, China. After this date, the number of patients increased rapidly, and the disease was observed common all over the world. A number of studies of the total burden of novel COVID-19 have demonstrated that, although generally more dangerous in adults at risk of COVID-19 related complications because of chronic underlying diseases such as cardiovascular and cerebrovascular diseases and diabetes (8).

COVID-19 is also seen common in otherwise healthy children, a considerable number of whom experience severe disease leading to hospitalisation, increased outpatient visits and pharmaceutical medication prescriptions, and (although rarely) even death (9). The first COVID-19 infected child patient in the world was reported from Shenzen on January 20, 2020 (10).

The management of the disease is not very clear and no reliability and effectiveness-proven treatment are yet available for COVID-19. The drugs used to treat it frequently cause adverse effects. Although there are previous studies on possible prognostic drugs in the treatment of pediatric COVID-19, there is no consensus for treatment (11-17).

To our knowledge, there is no major published study on pediatric COVID-19 in Turkish children. Therefore, in our study, we aimed to evaluate the clinical, radiological and laboratory features of the disease in Turkish children. Another objective of this retrospective study was to compare the pediatric COVID-19 disease characteristic in Turkey to those found in other countries. In our study, we also aimed to identify risk factors associated with coronavirus in Turkish children.

\section{Material and Methods}

This study included 105 pediatric patients between the ages of 0-18 years, who were diagnosed with COVID19 in Dicle University, Faculty of Medicine, Department of Pediatric which is the main center for management of pediatric Covid-19 patients in southeastern Turkey, between April 2020 and June 2020. The diagnosis of COVID-19 was made by the method of real-time reverse transcription-polymerase chain reaction (RT-PCR) in samples obtained with nasopharyngeal swab. During the diagnosis, BUN, creatinine, Alanine amino transferase (ALT), Aspartate amino transferase (AST), Lactate dehydrogenase (LDH), serum electrolytes, Prothrombin time (PT), Activated partial thromboplastin time (aPTT), International normalized ratio (INR), C-reactive protein (CRP), procalcitonin, troponin I, CK-MB and D-Dimer were studied in all patients. Posterior-anterior (PA) chest radiography was performed in every patient included in the study and in cases of pathological findings on radiography or clinically severe cases. The data that include patient demographics, laboratory and clinical results, and the previous treatments of the patients were retrospectively taken from patient files and computer-based patient records.

The severity of the disease was classified as asymptomatic, mild, moderate, severe, and critical according to the clinical characteristic, laboratory results, and chest radiography findings (18).

- Asymptomatic: Cases with a positive RT-PCR test without any clinical and radiological findings

- Mild: Cases with upper respiratory tract infection symptoms such as fever, fatigue, myalgia, cough, sore throat, nasal flow with normal espiratory system examination

- Moderate: Cases with pneumonia with complaints of fever and cough but without the symptoms of dyspnea and hypoxemia or cases with findings of COVID-19 on chest CT scan without any symptoms

- Severe: Cases with fever and cough in the early period who develop dyspnea and central cyanosis within a week (arterial oxygen saturation of $<92 \%$ )

- Critical: Cases who develop acute respiratory distress or respiratory failure rapidly, and who tend to develop shock, encephalopathy, myocardial affection, coagulation dysfunction, and acute kidney injury

The patients were divided into three age groups and the data were compared. These groups were as follows: Group 1, patients aged 0 to 1 year (0-12 months); Group 2, patients aged 1 to 10 years (13-120 months); 
Group 3, patients aged 10 to 18 years (130-216 months).

Patients over 18 years of age and suspected of having COVID-19 with clinical, laboratory, and radiological findings, but with negative RT-PCR test results were excluded from the study.

The approval was taken from the Republic of Turkey Ministry of Health and the Ethics Committee of Dicle University, Faculty of Medicine.

\section{Statistical Analysis}

Data analyses were examined by using Statistical Package for Social Sciences (SPSS), Version 20.0 for Windows (SPSS Inc., Chicago, IL, USA). The variables were investigated using visual (histograms, probability plots) and analytical methods (Kolmogorov-Simirnov test) whether or not they were normally distributed. Normally distributed variables were shown by using means and standard deviations, and non-normaly distributed variables were shown by using median and range (maximum and minimum). The chi-square test was used to analyze of categorical variables. Correlations of parameters were studied using Pearson or Spearman correlations. Multiple groups were compared using one-way analysis of variance (ANOVA) with a post-hoc Bonferroni correction and Kruskal-Wallis test for the normally and non-normally distributed variables, respectively. The independent variables for duration of hospital stay were investigated by using stepwise multiple linear regression analysis. $\mathrm{P}$ values $<0.05$ were considered statistically significant.

\section{Results}

The mean age of the patients was $108.64 \pm 65.61$ months (1.5 months-18 years). Of the patients, $51.4 \%$ (54/105) were male. There were 13 patients in Group 1, 46 patients in Group 2, and 46 patients in Group 3. The most common cause of transmission in pediatric patients was contacting with a family member diagnosed with COVID-19 ( $\mathrm{n}=91,86.7 \%)$.

Four of our patients $(3.8 \%)$ had an accompanying disease at the time of diagnosis. A 16-year-old patient had more than one comorbid factor (Diabetes Mellitus + Beta thalassemia major + chronic respiratory disease). A 5.5-month-old patient was followed-up for Primary Hemophagocytic lymphohistiocytosis before the diagnosis. One of whom with Down syndrome and Acute lymphoblastic leukemia, and the other with the diagnosis of epilepsy and was on anti-epileptic drugs.

The most common admission complaints were dry cough $(\mathrm{n}=17,16.2 \%)$, fever $(\mathrm{n}=16,15.2 \%)$, lassitude and fatigue $(\mathrm{n}=14,13.3 \%)$ and headache $(\mathrm{n}=12,11.4 \%)$, respectively. The admission complaints of the patients were summarized in Table 1.

Chest CT was performed on 48 patients. Pulmonary ground-glass opacities ( $\mathrm{n}=11,10.4 \%)$ were the most common chest CT findings detected in these patients (Fig. 1, 2 ). Other common findings were local patchy shadow $(\mathrm{n}=5,4.7 \%)$ and bilateral patchy shadow $(\mathrm{n}=4,3.8 \%)$, respectively. Pleural effusion was also seen in one of our patients.

A significant number of patients were clinically asymptomatic $(n=59,56.2 \%)$. Three patients required intensive care. The classification based on clinical severity was summarized in Table 1 . The mean length of hospital stay was 4 (range 1-19) days in the patients who required hospitalization. No mortality was observed in any of our patients.

Antibiotics and antiviral agents were used to treat the patients. The most commonly used drugs were the combinations of azithromycin $(\mathrm{n}=72,68.6 \%)$, hydroxychloroquine $(\mathrm{n}=21,20 \%)$, antibiotics $(\mathrm{n}=10,9.5 \%)$, oseltamivir $(n=8,7.6 \%)$, lopinavir-ritonavir $(n=4,3.8 \%)$. Only 3 of our patients required the use of oxygen. High flow oxygen was administered to a patient who required intensive care.

The clinical and laboratory findings of the patients were summarized in Table 2.

According to the differences between Groups 1, 2, and 3, the contact history was significantly lower in Group 1 than in other two groups $(\mathrm{p}<0.001)$. The frequency of male gender was significantly higher in Group 2 
$(\mathrm{p}=0.032)$ than in other two groups. Besides, sore throat and cough complaints were significantly higher in Group 3 than in other two groups ( $\mathrm{p}=0.004, \mathrm{p}=0.008$ ) (Table 3 ).

The distribution of the clinical severity of the patients by age groups was shown in Table 4 .

The Wbc and D-dimer levels, lymphocyte, and platelet counts were significantly higher in the patients in Group 2 compared to other two groups ( $\mathrm{p}=0.007, \mathrm{p}<0.001, \mathrm{p} 0.001, \mathrm{p}=0.005)$. The creatinine levels were lower in the patients in Group 1 than in other two groups $(\mathrm{p}<0.001)$, and the blood urea levels were lower in the patients in Group 1 than in the patients in Group $3(\mathrm{p}=0.048)$. The parameters that showed significant differences among the groups regarding the evaluation of the laboratory findings were shown in Table 5 .

There were significant positive correlations between length of hospitalization and CRP, ferritin, fibrinogen, Body temperature $(\mathrm{r}=0.871, \mathrm{r}=0.400, \mathrm{r}=0.648, \mathrm{r}=0.585, \mathrm{p}<0.001, \mathrm{p}=0.021, \mathrm{p}<0.001, \mathrm{p}<0.001$, respectively) and negative correlations with oxygen saturation, $(\mathrm{r}=-0.765, \mathrm{p}<0.001)$. We modelled a multiple linear regression analysis with enter model to define the independent variables of duration of length of hospital stay. Gender, CRP, ferritin levels, fibrinogen, oxygen saturation were included into the analysis. CRP was only independently factor associated longer duration of hospitalization $(\mathrm{p}<0.001$, table 6$)$.

\section{Discussion}

Children can carry the heaviest burden of viral respiratory diseases. However, corona virus related infections also vary widely among pediatric individuals. Studies from China and America recommend, that children with COVID-19 might be less serious than in adults and that pediatric cases might knowledge various symptoms than do adults $(19,20)$; nevertheless, disease characteristics among pediatric patients in the Turkey have not been described. The aim of this study was to determine the epidemiological characteristics of pediatric COVID-19 patients during the first 3 months (April - June) of the disease that occur in Turkey.

Our study included positive pediatric cases confirmed with the RT-PCR method. The male/female ratio in COVID-19 varies between studies. In previous reports from China and America, boy outnumbered girls (11, 19- 21). The present study showed a male $(51.4 \%)$ predominance, similar to what has been described in the Chinese and American populations (22-24). In previous studies, ages ranged between 1 day and 18 years (19, $20,22)$. In our study, the mean age of the patients was $108.64 \pm 65.61$ months and the age range of patients is between 45 days to 18 years. These results suggests that all ages of childhood were sensitive to COVID-19.

In a Chinese study, the most common symptoms in children with COVID-19 were reported as fever and dry cough $(36 \%, 19 \%$, respectively) (22). Also in an another previous large-scale study, fever and cough were reported more frequently in pediatric COVID-19 cases $(56 \%, 54 \%$, respectively), too. In our study, the most common symptoms on admission were cough (16.2\%), fever (15.2\%), lassitude and fatigue (13.3\%). In American study the frequency of sore throat, headache, and diarrhea have been found to be quite less in pediatric patients (20). The present study found the symptoms including; sore throat, headache, diarrhea, loss of taste, anosmia and vomiting $(8 \%, 20 \%, 4 \%, 2 \%, 3 \%$ and $6 \%$ respectively). These findings are in line with other studies from other countries. These results suggests that children do not always have fever or cough as reported signs and symptoms.

Comorbidities were present in $3.8 \%$ of the patients. The comorbidities were as follows: diabetes mellitus, beta thalasemia major, chronic respiratory disease, Down syndrome, acute lymphoblastic leukemia and epilepsy (25).

A 17-year-old girl with insulin-dependent type one diabetes (HbA1C: 8.6) and bone marrow transplantation due to beta thalassemia major and who developed bronchiolitis obliterans presented to the emergency department with shortness of breath and two weeks of a cough. On physical examination, there was tachycardia and high fever. Also, there was bilateral crepitan ral and roncus in auscultation. Laboratory workup was significant for white blood count (WBC) of $7.74 \times 103 / \mu \mathrm{L}$ with $0.99 \%$ eosinophils, blood glucose of $220 \mathrm{mg} / \mathrm{dL}$, elevated C-reactive protein, and normal venous blood gas. Initial blood and sputum cultures with gram stain were negative. Computed tomography (CT) of the chest showed ground glass, peribronchial consolidation areas and mosaic pattern in both lungs (Fig. 3 ). Despite initial treatment with oseltamivir, azithromycin 
and hydroxychloroquine treatment, the patient developed respiratory distress, so that lopinavir-ritonavir and favipiravir were added to the treatment. The PCR tests for SARS-COV-2 on the 9th and 11th days of treatment were negative. The patient was discharged on the 17th day with healin.

A 6-month-old male patient infected with COVID-19 in April 2020, while receiving Hemophagocytic Lymphohistiocytosis (HLH) 2004 chemotherapy protocol with the diagnosis of familial (Genetic / Primary) HLH. In our patient, which was accompanied by defective perforin gene defect in primary HLH pathogenesis, COVID-19 infection with the presence of fever and hyperferritinemia, was evaluated in favor of reactivation and the patient was given both the HLH-2004 chemotherapy protocol treatment and COVID-19 therapy as recommended by the guidelines. Our patient improved clinically and in terms of laboratory test results at the end of the 15-day hospitalization period and was discharged. It should be remembered that COVID-19 can be seen with different clinical manifestations in the pediatric age group, and COVID-19 test should be recommended, especially in children with immunosuppression and fever.

In a previous report covering 2135 pediatric patients with COVID-19, the disease followed an asymptomatic, mild, moderate, severe and critical. Regarding the severity, $4.4 \%, 51.0 \%$, and $38.7 \%$ cases were diagnosed as asymptomatic, mild, or moderate, respectively (totally $94.1 \%$ of all cases) (19). In another study found that children with COVID-19 had milder clinical manifestations and nearly half of pediatric patients were asymptomatic (22). In our study, $56.2 \%$ of the cases were asymptomatic, $30.5 \%$ were mild, $10.5 \%$ were moderate, $2.9 \%$ were severe and none of our patients were critical. The percentage of the patients requiring ICU admission was $2.9 \%$.

Laboratory findings in pediatric COVID-19 patients are generally similar to those in other coronavirus infections. The number of white cells is often normal or low; neutropenia and / or lymphopenia may accompany. Thrombocytopenia may develop. C reactive protein and procalcitonin values are generally normal. In severe cases, liver enzymes and lactate dehydrogenase may increase, and abnormal coagulation and high D-dimer levels have been reported in these cases (26). In our study, we found the elevated level of lactate dehydrogenase and D-dimer in 3 cases with severe disease.

Thoracic tomography findings in children are bilateral multiple patchy, nodular ground glass opacities and / or infiltrations in the middle and outer zones of the lung or under the pleura. In the present study, pulmonary ground glass opacities $(\mathrm{n}=11,10.4 \%)$ were found as the most common finding in chest tomography. Other common findings were; local patchy shadow $(\mathrm{n}=5,4.7 \%)$ and bilateral patchy shadow $(\mathrm{n}=4,3.8 \%)$, respectively. In one of our patients, pleural effusion was seen. These results are consistent with other reports $(11,21,27)$. The radiological findings are non-specific and milder than adults $(17,21,27,28)$.

To date, there are no published controlled clinical trials on pediatric COVID-19 specific drug therapy. As with other age groups, there is insufficient evidence for any drug that can be used in the treatment of COVID-19 in children. Therefore, suggested treatments for COVID-19 in child patients should be evaluated in accordance with the studies on adults and should be planned in specific to a child patient. World Health Organization and the American Center for Disease Control and Prevention does not recommend a specific drug for the treatment of children with COVID-19 $(29,30)$. In our country, the management of pediatric patients with COVID-19 is also evaluated by the Scientific Board of Ministry of Health at frequent intervals and revisions are made $(31,32)$. Probable adverse effects of drugs must be taken into consideration in the decision for treatment in child patients. Treatment should be evaluated in specific to each child patient, and medication may be planned for patients with probable severe pneumonia and mild cases with risk factor. Medicines used in the treatment of patients, respectively; azithromycin $(\mathrm{n}=42,40 \%)$, hydroxychloroquin $(\mathrm{n}$ $=21,20 \%)$, empirical antibiotic $(\mathrm{n}=10,9.5 \%)$, oseltamivir $(\mathrm{n}=8,7.6 \%)$, lopinavir-ritonavir $(\mathrm{n}=4,3.8 \%)$ and Favipiravir $(\mathrm{n}=1,0.9 \%)$. Only 3 patients needed oxygen therapy. High flow humidified oxygen was applied to a patient who was followed up in the intensive care unit.

There were some limitations to our study. First, this study was hospital-based, second it is possible the data may be incomplete and incorrect due to the retrospective study design.

\section{Conclusion}


To our knowledge, this study is the largest reported cohort of children with pediatric patients with COVID19 in Turkey. A clear understanding of the local epidemiology of corona virus infections and identification of risk factors is critical for the successful implementation of the prevention and control program. Long and prospective studies are needed to provide a clear understanding of the local epidemiology of respiratory tract infections and identification of risk factors, the successful implementation of a prevention and control program, and to clarify the role of respiratory pathogens.

\section{Conflicts of interest}

The authors report no conflicts of interest. The authors alone are responsible for the content and writing of the paper.

\section{References}

1. Dewald Schoeman and Burtram C. Fielding. Coronavirus envelope protein: current

Knowledge. Virology Journal.(2019) 16:69

2. Lu R., Zhao X., Li J., Niu P., Yang B., Wu H. Genomic characterisation and epidemiology of 2019 novel coronavirus: implications for virus origins and receptor binding. Lancet. 2020

3. Chen Y, Liu Q, Guo D. Emerging coronaviruses: genome structure, replication, and pathogenesis. J Med Virol. 2020;92:418-423.

4. Wu A, Peng Y, Huang B, Ding X, Wang X, Niu P et al (2020) Genome composition and divergence of the novel coronavirus (2019-nCoV) originating in China. Cell Host Microbe.

5. Paules CI, Marston HD, Fauci AS (2020) Coronavirus infections-more than just the common cold. JAMA.

6. Gao H, Yao H, Yang S, Li L. From SARS to MERS: evidence and speculation. Front Med. 2016;10:377-82

7. de Wit E, van Doremalen N, Falzarano D, Munster VJ. SARS and MERS: recent insights into emerging coronaviruses. Nat Rev Microbiol. 2016;14:523-534.

8. Chen N., Zhou M., Dong X., Qu J., Gong F., Han Y. Epidemiological and clinical characteristics of 99 cases of 2019 novel coronavirus pneumonia in Wuhan, China: a descriptive study. Lancet. 2020

9. Harapan H, Naoya I, Amanda Y, Wira W, S Keam, Haypheng Te. Coronavirus disease 2019 (COVID-19): A literature review. J Infect Public Health. 2020 May; 13(5): 667-673.

10. Chan JF, Yuan S, Kok KH, To KK, Chu H, Yang J, et al. A familial cluster of pneumonia associated with the 2019 novel coronavirus indicating personto-person transmission: a study of a family cluster. Lancet 2020;395:514-

11. Lu X, Zhang L, Du H, et al. SARS-CoV-2 infection in children. N Engl J Med.2020;382(17):1663-1665

12. Wang XF, Yuan J, Zheng YJ, et al. Novel coronavirus infection in 34 children in Shenzhen: clinical and epidemiological characteristics. Chin J Pediatr. 2020;58(0)

13. Cai J, Xu J, Lin D, et al. A case series of children with 2019 novel coronavirus infection: clinical and epidemiological features. Clin Infect Dis. 2020

14. Liu W, Zhang Q, Chen J, et al. Detection of Covid-19 in children in early January 2020 in Wuhan, China. N Engl J Med. 2020;382(14): 1370-1371.

15. Zhang YH, Lin DJ, Xiao MF, et al. [2019-novel coronavirus infection in a three-month-old baby]. Chin J Pediatr. 2020;58

16. Zhao R, Xu K. 2019 novel coronavirus infection in 1 children. Zhejiang Med J. 2020;42(4):305-306 
17. Zheng F, Liao C, Fan Q, et al. Clinical characteristics of children with coronavirus disease 2019 in Hubei, China. Curr Med Sci. 2020;40(2): 275-280.

18. Shen K, Yang Y, Wang T, et al. Diagnosis, treatment, and prevention of 2019 novel coronavirus infection in children: experts' consensus statement World J Pediatr. 2020;1-9.

19. Yuanyuan Dong, Xi Mo, Yabin Hu, Xin Qi, Fan Jiang, Zhongyi Jiang, Epidemiology of COVID-19 Among Children in China. Pediatrics, 2020:145, 6.

20. Stephanie Bialek, Ryan Gierke, Michelle Hughes, Lucy A. McNamara,corresponding author Tamara Pilishvili, and Tami Skoff. Coronavirus Disease 2019 in Children - United States, February 12-April 2, 2020. MMWR Morb Mortal Wkly Rep. 2020 Apr 10; 69(14): 422-426.

21. Ma Y-L, Xia S-Y, Wang M, Zhang S-M, Du W-H, Chen Q. Clinical features of children with SARS-CoV-2 infection: an analysis of 115 cases. Chin J Contemp Pediatr. 2020;22(4):290-293.

22. Haiyan Qiu, Junhua Wu, Liang Hong, Yunling Luo, Qifa Song, Dong Chen. Clinical and epidemiological features of 36 children with coronavirus disease 2019 (COVID-19) in Zhejiang, China: an observational cohort study. Lancet Infect Dis 2020 Jun;20(6):689-696.

23. Zhonghua Liu Xing Bing Xue Za Zhi. The Novel Coronavirus Pneumonia Emergency Response Epidemiology Team. The epidemiological characteristics of an outbreak of 2019 novel coronavirus diseases (COVID-19) -China, 2020. China CDC Weekly. 2020; 2(8):113-122

24. Guan WJ, Ni ZY, Hu Y, et al; China Medical Treatment Expert Group for COVID-19. Clinical characteristics of coronavirus disease 2019 in China.

N Engl J Med2020 Apr 30;382(18):1708-1720.

25. Lara S. Shekerdemian, MD, MHA1; Nabihah R. Mahmood, MD2; Katie K. Wolfe, MD3; et al. Characteristics and Outcomes of Children With Coronavirus Disease 2019 (COVID-19) Infection Admitted to US and Canadian Pediatric Intensive Care Units JAMA Pediatr. 2020 May 11.

26. Zimmermann P, Curtis N. Coronavirus Infections in Children Including COVID-19. An Overview of the Epidemiology, Clinical Features, Diagnosis, Treatment and Prevention Options in Children. Pediatr Infect Dis J 2020;39:355-368.

27. Wang D, Ju XL, Xie F, et al. [Clinical analysis of 31 cases of 2019 novel coronavirus infection in children from six provinces (autonomous region) of northern China]. Chin J Pediatr. 2020;58(4):269-274

28. Feng C, Liu ZS, Zhang FR, et al. First case of severe childhood novel coronavirus pneumonia in China. Chin J Pediatr. 2020;58:E005.

29. World Health Organization. WHO interim guidance on clinical management of severe acute respiratory infection when novel coronavirus $(\mathrm{nCoV})$ infection is suspected. 2020. Available at: https://apps.who.int/iris/handle/10665/330893.

30. Centers for Disease Control and Prevention CfDCaP. Interim clinical guidance for management of patients with confirmed 2019 novel coronavirus $(2019-\mathrm{nCoV})$ infection. 2020. Available at: https://www.cdc.gov/coronavirus/2019-ncov/hcp/clinical-guidance-management-patients. html.

31. Covid-19 (Sars-Cov-2 Infection), Child Patient Management And Treatment (Study Of Scientific Board), The Republic Of Turkey Ministry Of Health, March 2020.

32. Covid-19 (Sars-Cov-2 Infectıon), Child Patient Management And Treatment (Study Of Scientific Board), The Republic Of Turkey Ministry Of Health, April 2020.

\section{Hosted file}


Tables.docx available at https://authorea.com/users/337197/articles/468342-evaluation-ofthe-novel-coronavirus-disease-in-turkish-children-preliminary-outcomes

\section{Figures}

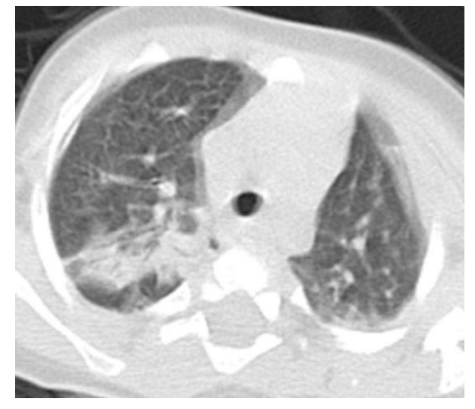

Figure 1. Bilateral ground glass and consolidation areas in a 7- month old girl with fever and cough

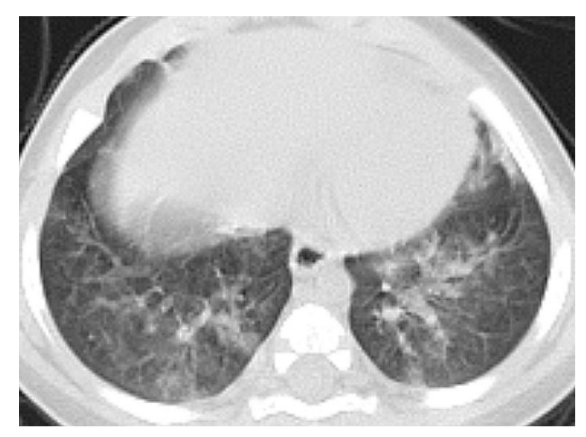

Figure 2. The CT image of a 9- month old girl with a history of fever, cough and close contact with a family member, revealing peripheral ground-glass areas in both lungs

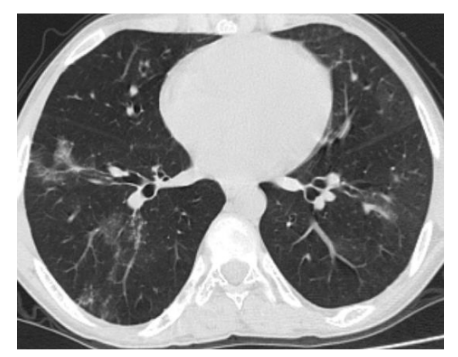

Figure 3. Computed tomography (CT) of a A 17-year-old girl with dyspnea and cough revealing ground glass and peribronchial consolidation areas 